\title{
Ribosomal DNA variation, recombination and inheritance in the basidiomycete Trichaptum abietinum: implications for reticulate evolution
}

\author{
$\mathrm{H}$ Kauserud and T Schumacher \\ Division of Botany and Plant Physiology, Department of Biology, University of Oslo, PO Box 1045, Blindern, N-0316 Oslo, Norway
}

\begin{abstract}
Two divergent nuclear ribosomal DNA (nrDNA) types, designated $\alpha$ and $\beta$, were found distributed in 11 North European populations of the basidiomycete Trichaptum abietinum. These types differed by a $220 \mathrm{bp}$ indel in the internal transcribed spacer 1 (ITS1) sequence and a number of linked substitutions and small indel motives in the internal transcribed and intergenic spacers (ITS1, ITS2, IGS1 and IGS2). The $\alpha$ and $\beta$ haplotypes co-occurred in heterozygous somatic individuals (dikaryons) and segregated in a Mendelian fashion in monokaryotic single spore progenies. This result suggests that the haplotypes are encoded in different nuclei of field-collected dikaryons and inherited as a single locus. No meiotic recombinants were observed among the sequenced monokaryons. Population genetic analyses by
\end{abstract}

PCR-RFLP revealed that a low frequency of evolutionary intermediate nrDNA types also existed in natural populations, presumably as a result of meiotic recombination of $\alpha$ and $\beta$ nrDNA. The existence of divergent nrDNA types in $T$. abietinum could be a result of a former independent evolution followed by a hybridization event. Phylogenetic analyses of ITS sequences suggest that the sister taxon $T$. fuscoviolaceum has been involved in the evolutionary history of $T$. abietinum. Sequence polymorphisms observed in the translation elongation factor $1 \alpha$ (efa) and glyceraldehyde-3phosphate dehydrogenase $(g p d)$ genes, did not reveal two well-defined types of these genes. The results are discussed in the light of other evolutionary mechanisms as well. Heredity (2003) 91, 163-172. doi:10.1038/sj.hdy.6800294

Keywords: Trichaptum abietinum; basidiomycota; nrDNA; reticulate evolution; meiotic segregation; recombination

\section{Introduction}

The genetic composition and structure within species, populations and individuals reflect their evolutionary history and may indicate which evolutionary mechanisms have been in action. Divergent speciation, mainly caused by mutations, selection and genetic drift, results in differentiated gene lineages. Reticulate evolution, that is, speciation via hybridization, combines divergent DNA lineages and is well documented among plants. Hybrid speciation has recently also been postulated as a track to rapid evolution in a few mutualistic and plant pathogenic fungi (Tsai et al, 1994; Garbelotto et al, 1996; O'Donnell and Cigelnik, 1997; O'Donnell et al, 1998; Brasier et al, 1999; Newcombe et al, 2000; Nielsen and Yohalem, 2001). Several studies have pointed out the usefulness of nuclear ribosomal DNA (nrDNA) sequence data in tracking the history of a species, including reticulate evolution (Sang et al, 1995; Wendel et al, 1995; O'Donnell and Cigelnik, 1997; O'Donnell et al, 1998; Brasier et al, 1999; Hughes and Petersen, 2000; Newcombe et al, 2000). The nuclear ribosomal gene family consists of reiterated cistrons of the ribosomal genes (18S, $5.8 S$ and 28S) interspersed by the internal transcribed and intergenic spacers (ITS and IGS). In plants, different

Received 25 June 2002; accepted 25 February 2003 outcomes have been reported for the ribosomal repeat following hybridization: (a) both parental ITS spacer sequences were retained (eg Franzke and Mummenhoff, 1999), (b) the ribosomal repeat was homogenized to one parental type (eg Wendel et al, 1995), (c) the ribosomal repeat was homogenized but contained scattered elements of both parents (eg Sang et al, 1995).

Trichaptum abietinum (Dicks.:Fr.) Ryvarden is a widespread wood-decaying fungus in boreal coniferous forests of the northern hemisphere (Ryvarden and Gilbertson, 1994). It can give rise to hundreds of small poroid basidiocarps (fruit bodies) on coniferous logs. The life history of the fungus is that of a typical basidiomycete, with a predominant dikaryotic vegetative state (each cell including two nuclei) and a short-lived monokaryotic state following meiosis before dikaryon formation. In some basidiomycetes, different intersterile populations (intersterility groups), which are fully or partially interfertile, have been observed. Intersterility groups (ISGs) generally equate to intraspecific units, and some differ in adaptive characters, for example those occurring within the root rot fungus Heterobasidion annosum (Garbelotto et al, 1996). T. abietinum comprises at least two North American ISGs that are partially compatible with a third European ISG (Macrae, 1967; Magasi, 1976). Four other Trichaptum species co-occur with T. abietinum on the northern hemisphere; T. fuscoviolaceum, which forms a toothed basidiocarp; T. laricinum, which forms a lamellate basidiocarp and T. biforme and T. subchartaceum, which form poroid basidiocarps 
(Gilbertson and Ryvarden, 1987; Ryvarden and Gilbertson, 1994). Phylogenetic analyses of ITS2 nrDNA and mtSSU sequences in Trichaptum have shown that the strains of each species formed monophyletic groups (Ko and Jung, 2002). However, ITS1 nrDNA sequence data subdivided the $T$. abietinum strains into three subgroups with $T$. fusco-violaceum included in one of the groups (Ko and Jung, 2002). It has been suggested that T. abietinum may be in the process of differentiation into new physiological or geographical taxa (Ko et al, 1997).

In this study, we report two divergent types of nuclear ribosomal repeat units in T. abietinum, designated $\alpha$ and $\beta$, as revealed by sequence and RFLP analyses of the nrDNA ITS and IGS regions in monokaryons and dikaryons from 11 populations of $T$. abietinum in Fennoscandia. To assess whether the observed nrDNA patterns were a result of reticulate evolution or other evolutionary mechanisms, we also examined partial sequences from the translation elongation factor $1 \alpha$ (efa) and glyceraldehyde-3-phosphate dehydrogenase $(g p d)$ genes and the mode of nrDNA inheritance in spore progenies. The results are discussed.

\section{Materials and methods}

\section{Sample}

In all, 249 basidiocarps from 11 populations of $T$. abietinum in coniferous forests of Fennoscandia were sampled in order to examine the geographic range of genetic variation at population level. Somatic cultures (dikaryons) were obtained from all basidiocarps. For sequencing, 10 single spore cultures (monokaryons) were obtained from germinating basidiospores of nine different fruit bodies from four populations. The isolates were grown on $2 \%$ PDA at $25^{\circ} \mathrm{C}$ in darkness. To determine whether the nrDNA polymorphisms are inherited in simple Mendelian ratios, we sampled $56(2 \times 28)$ single spore cultures (monokaryons), from two additional fruit bodies (TaGu1 and TaGu4), and performed a segregation experiment. The existence of somatic and single spore culture isolates relied on microscopic observation of respectively clamp connections on hyphae (dikaryons) or their absence (monokaryons).

\section{Molecular methods}

DNA was extracted by the CTAB miniprep method described by Murray and Thompson (1980) with some minor modifications: DNA was resuspended in $100 \mu \mathrm{l}$ sterile milliQ $\mathrm{H}_{2} \mathrm{O}$ at the final step of extraction, and DNA templates were diluted 50-100-fold before PCR amplification. Target sequences were amplified using the thermal profiles and primers given in Table 1 . The primer Ta300, positioned ca. $350 \mathrm{bp}$ upstream from the nuclear small subunit (18S) rRNA gene, was designed based on comparison with a partial sequence of the IGS2 in the material, which we derived from amplification with the primers 5SA' (Anderson and Stasovski, 1992) and CNS1 (White et al, 1990). The primer pairs EF595F/EF1160R (Kauserud and Schumacher, 2001), and EF1160F/ EF1750R (this study), were designed based on comparison with an accessioned efa sequence of Schizophyllum commune Fr. in GenBank (EMBL accession number X94913) (Table 1). PCR was performed in $40 \mu$ reactions containing $23.5 \mu \mathrm{l} 50 \times$ diluted template DNA and $16.5 \mu \mathrm{l}$ reaction mix (final concentrations: $4 \times 250 \mu \mathrm{M}$ dNTPs, $0.625 \mu \mathrm{M}$ of each primer, $2 \mathrm{mM} \mathrm{MgCl}_{2}$ and 1 unit DyNazyme $^{\mathrm{TM}}$ II DNA polymerase (Finnzymes Oy, Espoo, Finland) on a Genius Operator (Techne). Monokaryon amplicons were sequenced manually using the PCR primers, employing the ThermoSequenase radiolabelled terminator cycle sequencing kit (Amersham Pharmacia

Table 1 Primers and thermal profiles employed for PCR amplification of target sequences

\begin{tabular}{|c|c|c|c|c|c|}
\hline \multirow[t]{2}{*}{ Target sequence } & \multirow[t]{2}{*}{ Primer pairs } & \multirow[t]{2}{*}{ References } & \multicolumn{3}{|c|}{ Thermal profile } \\
\hline & & & Denat. & Repeated part & Exten. \\
\hline \multirow[t]{2}{*}{ ITS } & ITS5 & White et al (1990) & $94^{\circ} \mathrm{C}$ & $94^{\circ} \mathrm{C}-54^{\circ} \mathrm{C}-72^{\circ} \mathrm{C}$ (36 rep) & $72^{\circ} \mathrm{C}$ \\
\hline & ITS4 & White et al (1990) & $4 \mathrm{~min}$ & $30 s-35 s-40 s$ & $10 \mathrm{~min}$ \\
\hline \multirow[t]{2}{*}{ ITS2 } & ITS3 & White et al (1990) & $94^{\circ} \mathrm{C}$ & $94^{\circ} \mathrm{C}-52^{\circ} \mathrm{C}-72^{\circ} \mathrm{C}$ (36 rep) & $72^{\circ} \mathrm{C}$ \\
\hline & ITS4 & White et al (1990) & $4 \mathrm{~min}$ & $30 s-35 s-40 s$ & $10 \mathrm{~min}$ \\
\hline \multirow[t]{2}{*}{ IGS1 } & CNL12 & White et al (1990) & $94^{\circ} \mathrm{C}$ & $94^{\circ} \mathrm{C}-55^{\circ} \mathrm{C}-72^{\circ} \mathrm{C}$ (36 rep) & $72^{\circ} \mathrm{C}$ \\
\hline & 5SA & Anderson and Stasovski (1992) & $4 \mathrm{~min}$ & $30 s-35 s-40 s$ & $10 \mathrm{~min}$ \\
\hline \multirow[t]{2}{*}{ IGS2 } & $5 S A^{\prime}$ & Anderson and Stasovski (1992) & $94^{\circ} \mathrm{C}$ & $94^{\circ} \mathrm{C}-58^{\circ} \mathrm{C}-72^{\circ} \mathrm{C}$ (37 rep) & $72^{\circ} \mathrm{C}$ \\
\hline & CNS1 & White et al (1990) & $4 \mathrm{~min}$ & $30 s-35 s-40 s$ & $10 \mathrm{~min}$ \\
\hline \multirow[t]{2}{*}{ IGS2-segment } & Та300 & This study & $94^{\circ} \mathrm{C}$ & $94^{\circ} \mathrm{C}-55^{\circ} \mathrm{C}-72^{\circ} \mathrm{C}$ (37 rep) & $72^{\circ} \mathrm{C}$ \\
\hline & CNS1 & White et al (1990) & $4 \mathrm{~min}$ & $30 s-35 s-40 s$ & $10 \mathrm{~min}$ \\
\hline \multirow[t]{2}{*}{ gpd segment } & CTK-052 & Kreuzinger et al (1996) & $94^{\circ} \mathrm{C}$ & $94^{\circ} \mathrm{C}-55^{\circ} \mathrm{C}-72^{\circ} \mathrm{C}(37 \mathrm{rep})$ & $72^{\circ} \mathrm{C}$ \\
\hline & CTK-032 & Kreuzinger et al (1996) & $4 \mathrm{~min}$ & $30 s-35 s-40 s$ & $10 \mathrm{~min}$ \\
\hline \multirow[t]{2}{*}{ efa part 1} & EF595F & Kauserud and Schumacher (2001) & $94^{\circ} \mathrm{C}$ & $94^{\circ} \mathrm{C}-55^{\circ} \mathrm{C}-72^{\circ} \mathrm{C}(37 \mathrm{rep})$ & $72^{\circ} \mathrm{C}$ \\
\hline & EF1160R ${ }^{c}$ & Kauserud and Schumacher (2001) & $4 \mathrm{~min}$ & $30 s-35 s-40 s$ & $10 \mathrm{~min}$ \\
\hline \multirow[t]{2}{*}{ efa part 2} & $\mathrm{EF} 1160 \mathrm{~F}^{\mathrm{d}}$ & This study & $94^{\circ} \mathrm{C}$ & $94^{\circ} \mathrm{C}-55^{\circ} \mathrm{C}-72^{\circ} \mathrm{C}$ (37 rep) & $72^{\circ} \mathrm{C}$ \\
\hline & EF1750R & This study & $4 \mathrm{~min}$ & $30 s-35 s-40 s$ & $10 \mathrm{~min}$ \\
\hline
\end{tabular}

ePrimer sequence $\left(5^{\prime}-3^{\prime}\right)$ : GTACTCGTTGTAGGACTCAA. 
Biotech Inc., OH, USA) and $\alpha-{ }^{33}$ P-ddNTPs. EMBL/ GenBank/DDBJ accession numbers of sequences are: AJ309808-AJ309815 for the divergent ITS1, IGS1, IGS2 and ITS2 haplotypes, AJ309882-AJ309891 for the five efa haplotypes (two parts) and AJ309892-AJ309901 for the five gpd haplotypes (two parts). Identical nrDNA spacer sequences were obtained on complementary strands and in isolates from different populations, ruling out the possibility of PCR or sequencing artifacts. For restriction analyses, $10 \mu \mathrm{l}$ of ITS2 and IGS2 PCR products were digested in $25 \mu \mathrm{l}$ volumes containing $12 \mu \mathrm{l} \mathrm{H}_{2} \mathrm{O}, 2.5 \mu \mathrm{l}$ buffer and $0.5 \mu \mathrm{l} R s a \mathrm{I}$ and $D d e \mathrm{I}$, respectively, following the manufacturer's instructions (Promega, Southampton). Restriction products were separated on a $2 \%$ agarose gel stained with ethidium bromide, using 0.5 TBE as the running buffer, and photographed over UV light.

\section{Sequence alignments and phylogenetic analyses}

Available ITS nrDNA sequences from Trichaptum spp. were retrieved from GenBank (Table 2) and aligned in BioEdit (Hall, 1999) together with sequences obtained in this study. One large indel region of the ITS1 alignment (characters 38-332) was excluded prior to analyses. Maximum parsimony analyses of the aligned ITS1 and ITS2 sequences were performed separately in PAUP* v. 4.02b (Swofford, 1999). Parsimony analyses were carried out using the branch-and-bound option with the following parameter settings: gaps treated as missing, equal character weights, MultTrees option in effect, maxtrees setting to 50000 and branches having minimum length zero allowed to collapse yielding polytomies. To examine the support for inferred relations, bootstrap analyses (1000 replicates) were performed (Felsenstein, 1985) using default options.

\section{Results}

\section{Sequence variation in the nrDNA spacers}

PCR amplification of the ITS nrDNA region (ITS1-5.8SITS2) in dikaryons of T. abietinum gave two amplicons of different lengths, designated $\alpha$ (596bp) and $\beta$ (818bp). These co-occurred in the same (heterozygotes) or in different (homozygotes) dikaryotic isolates of the geographic populations (Figure 1a).

Seven single spore isolates (monokaryons) from four different populations yielded amplicons of either the $\alpha$ or $\beta$ type, each isolate possessing a single ITS sequence of $\alpha$ or $\beta$ type. Four monokaryons derived from the populations of Skotjernfjell and Kuhmo possessed the ITS $\beta$ type, and three monokaryons from the populations of Lierne, Umeå and Skotjernfjell possessed the ITS $\alpha$ type (Figure 2a). A $220 \mathrm{bp}$ indel, positioned $29 \mathrm{bp}$ downstream in the aligned ITS1, and seven minor indels and substitutions consistently distinguished the $\alpha$ and $\beta$ ITS types into two main ITS1 types, designated ITS1 $\alpha$ (239 bp) and ITS1 $\beta$ (461 bp) (Figure 2a). Substitutions in positions $113(\mathrm{~A} / \mathrm{G})$ and $189(\mathrm{GC} / \mathrm{AT})$ of the ITS2 (202 bp) distinguished two ITS2 types, designated ITS $2 \alpha$ and ITS2 $\beta$ (Figure 2a).

PCR amplification of the partial IGS1 in T. abietinum dikaryons gave two amplicons of different lengths, recognized as IGS1 $\alpha(407 \mathrm{bp})$ and IGS1 $\beta(410 \mathrm{bp})$ in the subsequently sequenced monokaryons. Indels at positions 358 and 374, recognized as single motif microsatellites (a G following six G's, and TT following $11 \mathrm{~T}^{\prime} \mathrm{s}$ ), distinguished the $\alpha$ and $\beta$ IGS1 sequences (Figure 2a). The single-banded partial IGS2 amplicons of T. abietinum dikaryons were resolved as two IGS2 haplotypes of the same length (352 bp), distinguished as IGS2 $\alpha$ and IGS2 $\beta$ based on indels at positions $26(-/ \mathrm{C})$ and $37(\mathrm{G} /-)$ upstream from the $18 \mathrm{~S}$ gene (Figure 2a). A sequence linkage was observed between the ITS/IGS $\alpha$ spacer versions, and between the ITS/IGS $\beta$ spacer versions. Thus, two distinct nrDNA types (linkage groups) were observed among the sequenced monokaryons (Figure 2a).

\section{Sequence variation in efa and gpd}

Sequence analyses of two amplified portions (505 and $480 \mathrm{bp}$ ) of the efa gene from seven monokaryons representing three populations, (Skotjernfjell, Kuhmo and Umeå), revealed 10 polymorphisms, which consti-

Table 2 GenBank sequences used in this study, published by Ko and Jung (2002)

\begin{tabular}{|c|c|c|c|c|c|}
\hline \multirow[t]{2}{*}{ Taxon } & \multirow[t]{2}{*}{ Strain number } & \multirow[t]{2}{*}{ Locality } & \multicolumn{3}{|c|}{ GenBank accession nos. } \\
\hline & & & ITS1 & & ITS2 \\
\hline T. abietinum & CBS347.68 & Canada & AF266676 & & AF267651 \\
\hline T. abietinum & CCFC008387 & Canada & AF266677 & & AF267652 \\
\hline T. abietinum & CBS357.68 & Canada & AF267648 & & AF266681 \\
\hline T. abietinum & ССFC009931 & Canada & AF267646 & & AF267653 \\
\hline T. abietinum & CCFC010156 & Canada & AF267647 & & AF267654 \\
\hline T. abietinum & SFC950815-17 & Korea & AF266680 & & AF267655 \\
\hline T. abietinum & SFC960608-11 & Korea & AF267644 & & AF267656 \\
\hline T. abietinum & SFC961028-11 & Korea & AF267645 & & AF267657 \\
\hline T. biforme & FP-86522 & USA & & U63476 & \\
\hline T. biforme & HНB-7316 & USA & & U63473 & \\
\hline T. fusco-violaceum & FP-133997 & USA & & U63472 & \\
\hline T. fusco-violaceum & HHB-4016 & USA & & U63478 & \\
\hline T. subchartaceum & CCFC003928 & Canada & AF267650 & & AF267659 \\
\hline T. subchartaceum & CCFC003932 & USA & AF266678 & & AF266679 \\
\hline T. laricinum & RLG-4665 & USA & & U63471 & \\
\hline T. laricinum & RLG-6936 & USA & & U63477 & \\
\hline
\end{tabular}




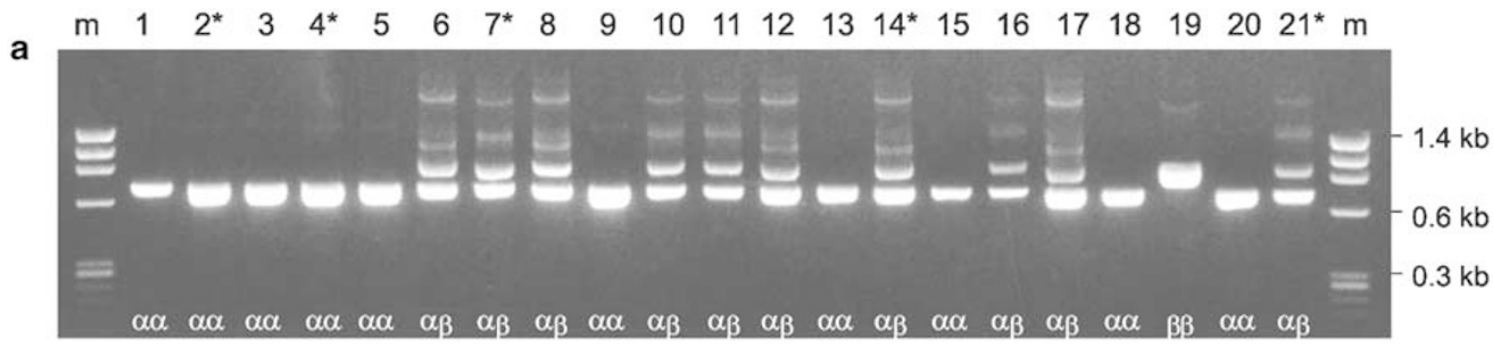

b

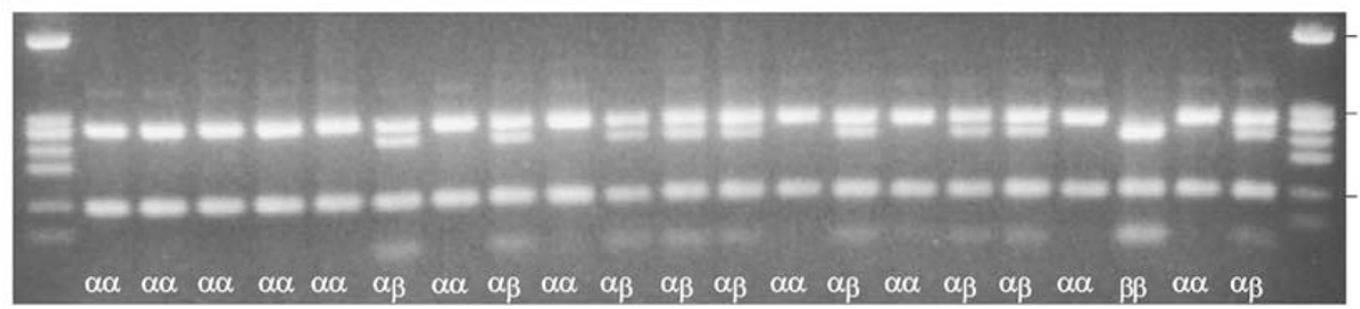

$0.6 \mathrm{~kb}$

$0.3 \mathrm{~kb}$

C

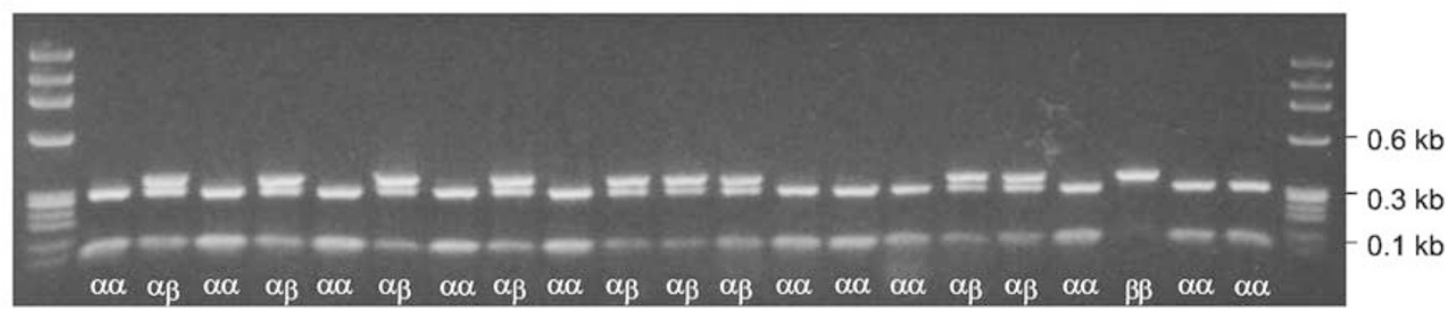

Figure 1 (a) Agarose gel showing ITS amplicons of 21 dikaryotic isolates from the Lierne population (Norway). Two distinct ITS products were found: a short version $(596 \mathrm{bp})$ and a long version $(818 \mathrm{bp})$, separated by a $220 \mathrm{bp}$ indel in ITS1. The two ITS products reflect the distribution of ITS1 $\alpha$ and $\beta$ versions. In heterozygous isolates including both versions (labelled $\alpha \beta$ ), the short version was favoured in the PCR reaction, giving a more prominent ITS1 $\alpha$ and a weaker ITS1 $\beta$. Artificial heteroduplex bands are also visible in heterozygous isolates. (b) The ITS2 region digested by RsaI. The different restriction patterns reflect the ITS2 $\alpha$ and $\beta$ types. (c) The partial IGS2 region digested by DdeI. The different restriction patterns reflect the IGS2 $\alpha$ and $\beta$ types. Five isolates $\left(2,4,7,14\right.$ and 21) show recombinant nrDNA genotypes (marked $\left.{ }^{*}\right)$ that possess recombined $\alpha$ and $\beta$ nrDNA spacer combinations. $m=$ size markers (X-174 DNA digested with HaeIII).

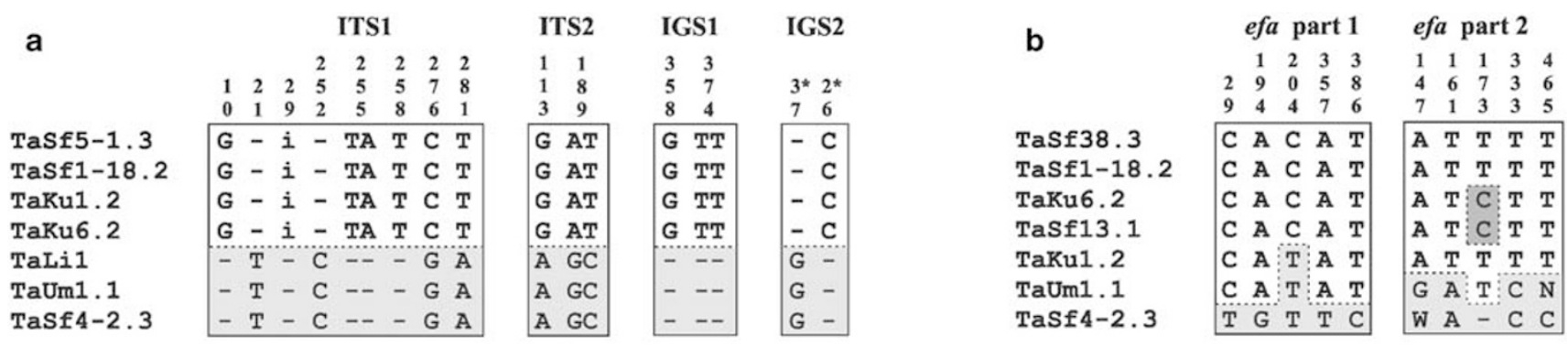

C

gpd part 1 $\begin{array}{llllllllllll}3 & 4 & 5 & 5 & 0 & 0 & 2 & 2 & 5 & 1 & 1 & 2\end{array}$ $\begin{array}{llllllllllll}6 & 2 & 5 & 8 & 4 & 8 & 3 & 9 & 6 & 0 & 8 & 3\end{array}$

Tasf 38.3 TaSf4-2.3 TaSf3.1

TaKu6. 2

TaKu1. 2

TaSf1-18.2
T A T A C T C T C T C A TCG A A A C T G C A T A G ANT G

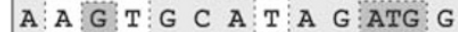
A $A$ C $T: G$ C A G A G ANT G $A$ A C $G$ G C A G A G ANT G gpd part 2

$\begin{array}{lllllllllllllllllllllllllllll}1 & 1 & 1 & 1 & 1 & 1 & 2 & 2 & 2 & 2 & 2 & 2 & 2 & 2 & 2 & 2 & 2 & 2 & 2 & 2 & 3 & 3\end{array}$ $\begin{array}{llllllllllllllllllllllllll}5 & 5 & 7 & 8 & 1 & 3 & 4 & 5 & 5 & 7 & 0 & 2 & 2 & 3 & 4 & 4 & 4 & 4 & 5 & 6 & 7 & 8 & 8 & 9 & 0 & 0\end{array}$ $\begin{array}{llllllllllllllllllllllllll}1 & 7 & 8 & 1 & 4 & 8 & 1 & 0 & 9 & 7 & 7 & 2 & 6 & 0 & 1 & 3 & 7 & 9 & 2 & 1 & 2 & 2 & 7 & 5 & 2 & 9\end{array}$

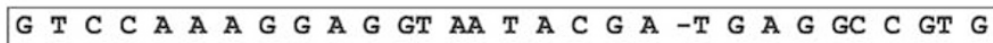
G T C C A A A G G A G GT AA T A C G A T: C G A A A A G G A G GT AA C A C G A T C G A A A A G G G G AT G C C A T T G

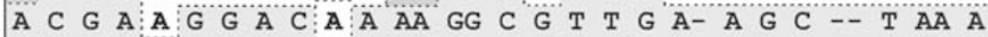
A C G A - G G A C A A AA GG C G T T G A-A G C - $A$ AA A

Figure 2 Distribution of sequence polymorphisms in target sequences: (a) the ITS1 (239/461 bp), ITS2 (202 bp), IGS1 (407/410bp) and the partial IGS2 nrDNA sequences ( $352 \mathrm{bp})$, (b) two amplified portions $(505+480 \mathrm{bp})$ of the efa gene and (c) sequenced $218+309 \mathrm{bp}$ portions of the gpd gene. Isolate codes are shown to the left in each matrix. The two first letters of isolate codes indicate taxon name (Ta $=T$. abietinum); the third and fourth letters indicate population (eg Um=Umeå), and the numbers refer to isolates. The position of the polymorphic sites in the aligned sequence matrices is written vertically above columns. The dotted line and grey shadings show the positions of sequence polymorphisms. In one position of efa (173) and in four positions of gpd, three different character states occurred among the haplotypes ( $\mathrm{i}=220 \mathrm{bp}$ indel in ITS1, ${ }^{*}=$ position upstream from the $18 \mathrm{~S}$ gene, $\mathrm{W}=\mathrm{A} / \mathrm{T}, \mathrm{N}=\mathrm{A} / \mathrm{C} / \mathrm{G} / \mathrm{T}$ ). 
tuted five distinct efa haplotypes (Figure $2 \mathrm{~b}$ ). The two most divergent efa haplotypes came from the same population (Skotjernfjell) and did not share any of the 10 polymorphisms. Two sequences from Skotjernfjell (TaSf38.3 and TaSf1-18.2) yielded the same efa haplotype, while a third sequence (TaSf13.1), constituting another efa haplotype, was identical to one of the sequences derived from the Kuhmo population (TaKu6.2). The three remaining efa sequences from Kuhmo, Umeå and Skotjernfjell yielded unique haplotypes. In one position (173, part 2) three character states (T, C or gap) occurred (Figure 2b).

A ca. $1 \mathrm{kbp}$ portion of the gpd gene was amplified from six monokaryotic single spore isolates representing two populations (Skotjernfjell and Kuhmo), and sequenced from each end. A total of 38 substitutions and minor indel motives appeared in the $549(218+309)$ bp long partial sequences of the $g p d$ gene, making up five distinct gpd haplotypes (Figure 2c). Two sequences from Skotjernfjell (TaSf38.3 and TaSf4-2.3) yielded identical gpd haplotypes. Two highly divergent gpd haplotypes (TaSf38.3 and TaSf1-18.2), derived from one of the populations, (Skotjernfjell) shared only two out of 38 polymorphic sites. The three remaining gpd haplotypes, derived from both populations, were evolutionary intermediate in sequence composition (see Figure 2c).

\section{ITS phylogenies}

Phylogenetic analyses of the 268-character ITS1 and 236character ITS2 data matrices (of which 57 and 65 characters were parsimony informative) gave three and four MPTs, 95 and 90 steps long (Figure 3). The ITS1 and ITS2 MPTs all recognized four groups of Trichaptum strains: one $T$. abietinum/T. fusco-violaceum clade, one $T$. biforme, one T. subchartaceum and one T. laricinum clade. In the ITS1 tree, there was support for various subgroups within the T. abietinum/fusco-violaceum clade and the $T$. fusco-violaceum strains were included as parts of one of the T. abietinum subclades ( $87 \%$ bootstrap support). On the contrary, the T. fusco-violaceum strains were placed at the base within the T. abietinum/T. fusco-violaceum clade in the ITS2 tree (Figure 3).

An alignment of the variable characters of the $T$. abietinum and T. fusco-violaceum ITS1 sequences, clearly demonstrated the existence of three ITS1 length types with sparse sequence variation in the worldwide sample of T. abietinum (Figure 4). The ITS1 $\alpha$ type of the North European T. abietinum strains is also present in three Canadian strains, and the $\beta$ type in three Korean strains. A third type, of intermediate length (425-431 bp), also occurred in T. abietinum and in two T. fusco-violaceum strains. The ITS1 $\alpha$ and $\beta$ types of the North European specimens shared five minor indels not observed in the other strains (Figure 4).

\section{nrDNA recombination}

Amplification of the ITS1 from 249 dikaryotic isolates, constituting all geographic populations (11), gave varying frequencies of the $\alpha$ and $\beta$ ITS1 nrDNA versions across populations (Table 3, Figure 5a). We used Webcutter 2.0 (Heiman, 1997; http://www.firstmarket. com/cutter/cut2.html), and found that the endonucleases RsaI and DdeI were able to digest and discriminate between the versions of the ITS2 and IGS2 sequences obtained, based on sequence polymorphisms in positions 113 (ITS2) and 37 (IGS2) (cf. Figure 2a). No suitable endonucleases were found for IGS1 restriction. In order to investigate whether the distribution of ITS2/
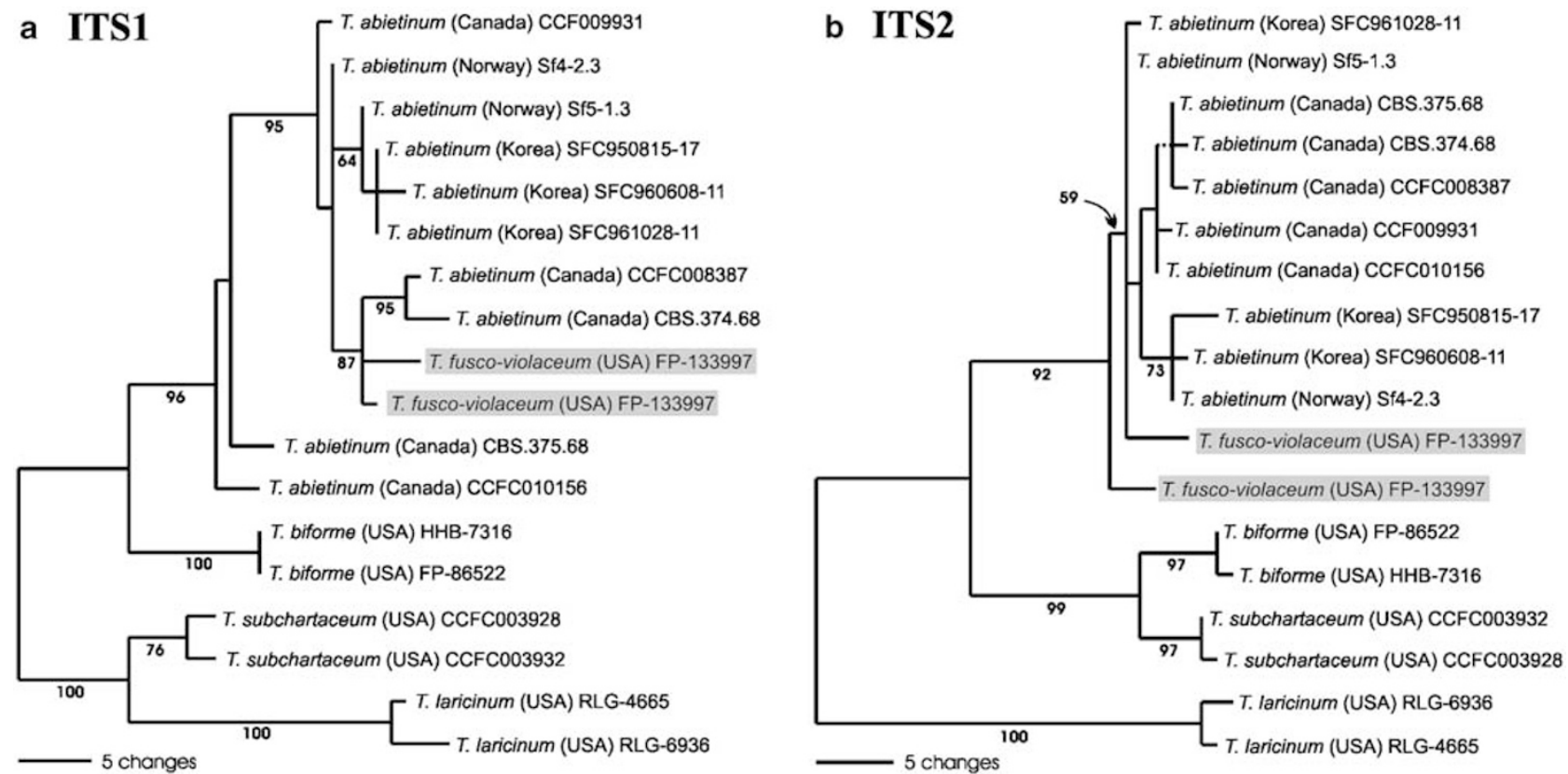

Figure 3 (a) ITS1 and (b) ITS2 phylogenies of Trichaptum specimens, obtained by use of the branch-and-bound search option in PAUP* v. $4.02 \mathrm{~b}$ (Swofford, 1999). Sequences are labelled with taxon names, geographical origin of specimens (in clamps) and collection names. (a) One out of three most parsimonious ITS1 phylograms, 95 steps long $(\mathrm{RI}=0.954, \mathrm{HI}=0.074)$. (b) One out of four most parsimonious ITS2 phylograms, 90 steps long $(\mathrm{RI}=0.911, \mathrm{HI}=0.089)$. Stippled lines collapsed in the strict consensus trees. Bootstrap support values $(>50 \%)$ are shown below nodes. 


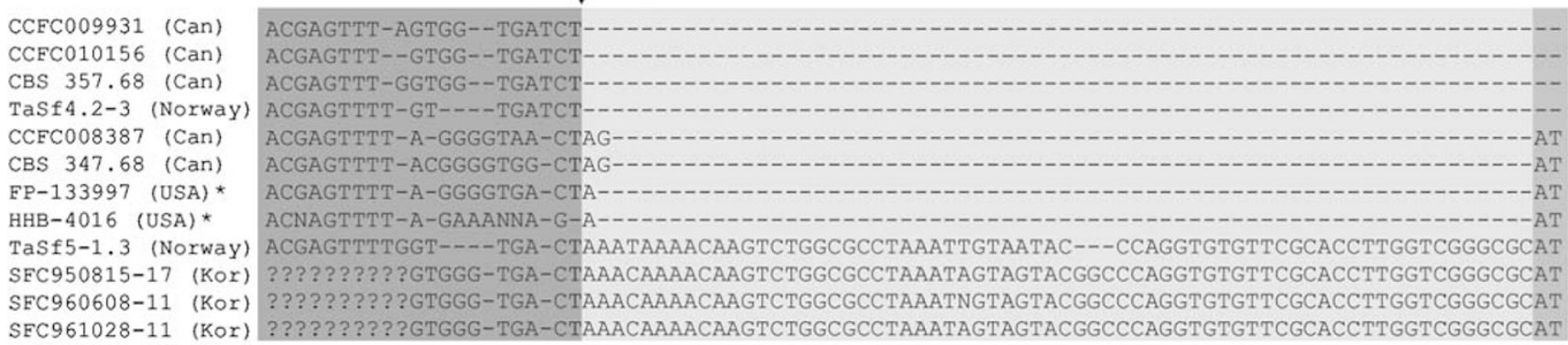

CCFC009931 (Can) CCEC010156 (Can) CBS 357.68 (Can) TaSf 4.2-3 (Norway) CCFC008387 (Can) CBS 347.68 (Can) FP-133997 (USA) * HHB -4016 (USA) * TaSf5-1.3 (Norway) SFC950815-17 (Kor) $\begin{array}{ll}\text { SFC } 960608-11 & \text { (Kor) } \\ \text { SFC961028-11 } & \text { (Kor) }\end{array}$

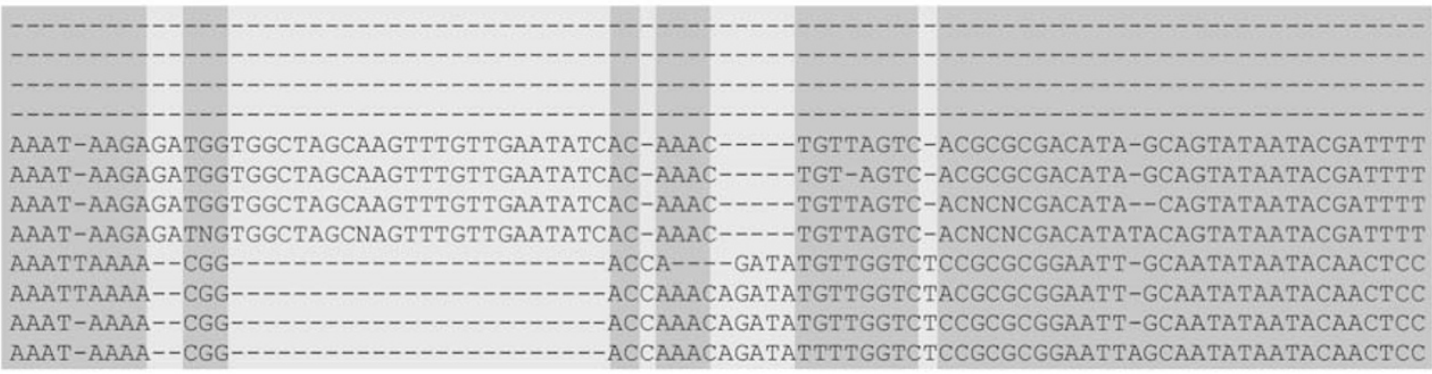

CCFC009931 (Can) CCFC 010156 (Can) CBS 357.68 (Can) TaSf 4.2-3 (Norway) CCFC008387 (Can) CBS 347.68 (Can) FP-133997 (USA)* HHB-4016 (USA) *

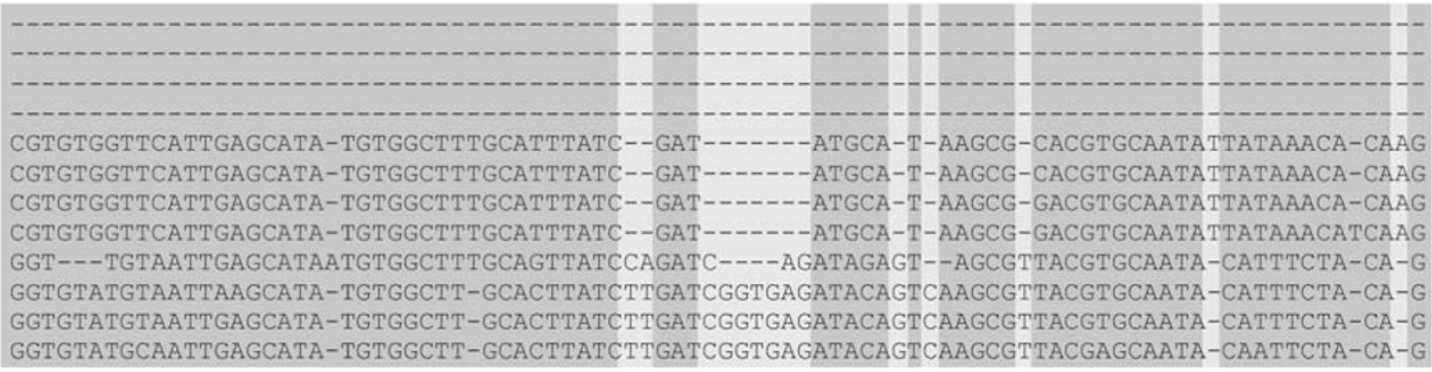
TaSf5-1.3 (Norway) GGT---TGTAATTGAGCATAATGTGGCTTTGCAGTTATCCAGATC----AGATAGAGT--AGCGTTACGTGCAATA-CATTTCTA-CA-G SFC950815-17 (Kor) GGTGTATGTAATTAAGCATA-TGTGGCTT-GCACTTATCTTGATCGGTGAGATACAGTCAAGCGTTACGTGCAATA-CATTTCTA-CA-G $\begin{array}{lll}\text { SFC960608-11 } & \text { (Kor) GGTGTATGTAATTGAGCATA-TGTGGCTT-GCACTTATCTTGATCGGTGAGATACAGTCAAGCGTTACGTGCAATA-CATTTCTA-CA-G } \\ \text { SFC961028-11 } & \text { (Kor) GGTGTATGCAATTGAGCATA-TGTGGCTT-GCACTTATCTTGATCGGTGAGATACAGTCAAGCGTTACGAGCAATA-CAATTCTA-CA- }\end{array}$

\section{CCFC009931 (Can) CCEC010156 (Can) CBS 357.68 (Can) TaSf $4.2-3$ (Norway) CCEC008387 (Can) CBS 347.68 (Can) $\mathrm{FP}-133997(\mathrm{USA})$ * HHB-4016 (USA)* TaSf5-1.3 (Norway)} SFC950815-17 (Kor) SFC960608-11 (Kor) TTT-CGGGTTGCGCGCGGGTCACTCAATAAT- SEC961028-11 (Kor) TTT-CGGGTTGCGCGCGGGTCACTCAATAAT

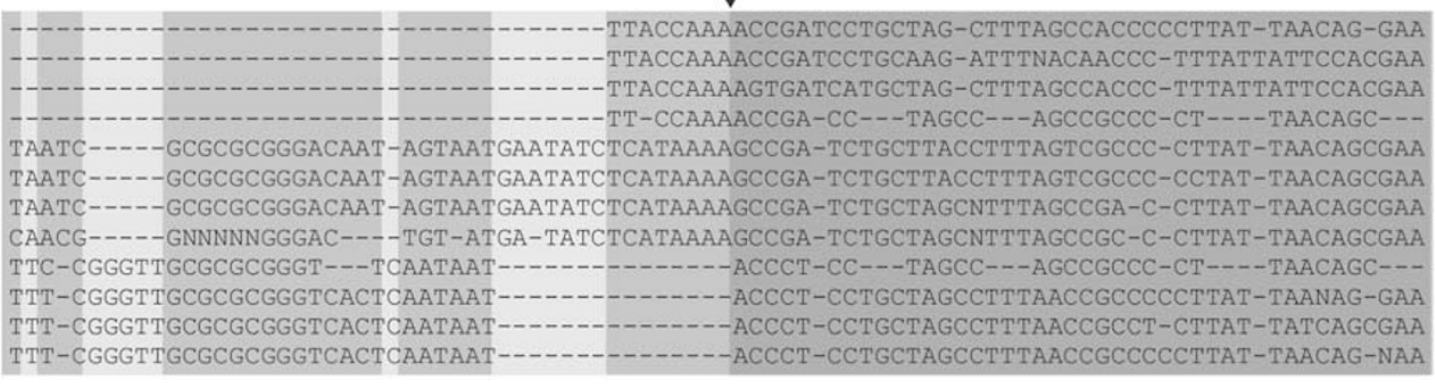

Figure 4 ITS1 sequence alignment of T. abietinum and T. fusco-violaceum (the latter indicated by *) including only variable characters. Three main ITS1 length types can be recognized. Dark grey shading indicates regions shared by all the three ITS1 length types, medium grey indicates regions shared by the two length types, and light grey indicates regions included in only one length type. The region between the arrows was deleted prior to phylogenetic analysis.

IGS2 $\alpha$ coalesced with the distribution of ITS1 $\alpha$, and ITS2/IGS2 $\beta$ with ITS1 $\beta$, we performed RsaI and DdeI restriction digest analyses of ITS2 and IGS2 amplicons of all dikaryotic isolates. Of the 249 dikaryons $85.5 \%$ (213) had combinations of solely ITS1/ITS2/IGS2 $\alpha$ or ITS1/ ITS2/IGS2 $\beta$ nrDNA versions, referred to here as nonrecombinant $\alpha$ or $\beta$ nrDNA; and $14.5 \%$ (36) showed intralocus recombinant nrDNA genotypes, possessing combinations of $\alpha$ and $\beta \operatorname{nrDNA}$ versions (cf. Figure 1, Table 3). The intralocus recombination ratios were $5.0 \%$ between ITS1 and ITS2, 10.1\% between ITS2 and IGS2 and $15.1 \%$ between ITS1 and IGS2. The proportion of nrDNA recombinants varied in the different populations.
No recombinants were observed in the Lammi population (Finland), whereas $12(52.2 \%)$ were observed in the Voss population (Norway) (cf. Table 3, Figure 5b). The proportion of $\alpha$ and $\beta$ spacer types varied overall from $58.8 \% \alpha$ in ITS1 to $61.6 \% \alpha$ in ITS2 to $65.1 \% \alpha$ in IGS2, with a proportional decline in the ITS1, ITS2 and IGS2 $\beta$ spacer types. A similar pattern was observed in 10 out of the 11 populations studied.

\section{Inheritance of nrDNA spacers}

Analysis of ITS1 $\alpha$ and $\beta$ distribution in a total of $2 \times 28$ single spore progenies (monokaryons), representing two 
Table 3 Distribution of ITS1 $\alpha / \operatorname{ITS} 1 \beta$, ITS $2 \alpha / \operatorname{ITS} 2 \beta$ and IGS2 $\alpha / \operatorname{IGS} 2 \beta$ in 11 populations of Trichaptum abietinum. Recombination frequencies between spacer versions are calculated

\begin{tabular}{|c|c|c|c|c|c|c|c|}
\hline \multirow[t]{2}{*}{ Population } & \multirow[t]{2}{*}{$n$} & \multicolumn{3}{|c|}{ Haplotype distribution (\%) } & \multicolumn{3}{|c|}{ Recombination (\%) } \\
\hline & & ITS1 $\alpha / \beta$ & ITS2 $\alpha / \beta$ & $\operatorname{IGS} 2 \alpha / \beta$ & ITS1-ITS2 & ITS2-IGS2 & ITS1-IGS2 \\
\hline Skotjernfjell & 26 & $48.1 / 51.9$ & $50.0 / 50.0$ & $51.9 / 48.1$ & 3.8 & 3.8 & 7.7 \\
\hline Sigdal & 33 & $61.4 / 38.6$ & $63.6 / 36.4$ & $68.2 / 31.8$ & 4.5 & 18.2 & 22.7 \\
\hline Lierne & 21 & $71.4 / 28.6$ & $73.8 / 26.2$ & $73.8 / 26.2$ & 4.8 & 19.0 & 23.8 \\
\hline Uppsala & 21 & $61.9 / 38.1$ & $61.9 / 38.1$ & $66.7 / 33.3$ & 0.0 & 9.5 & 9.5 \\
\hline Lammi & 21 & $73.8 / 26.2$ & $73.8 / 26.2$ & $73.8 / 26.2$ & 0.0 & 0.0 & 0.0 \\
\hline Grimstad & 19 & $57.9 / 42.1$ & $63.2 / 36.8$ & $65.8 / 34.2$ & 5.3 & 5.3 & 10.5 \\
\hline Voss & 23 & $41.3 / 58.7$ & $50.0 / 50.0$ & $69.6 / 30.4$ & 17.4 & 34.8 & 52.2 \\
\hline Umeå & 20 & $45.0 / 55.0$ & $45.0 / 55.0$ & $45.0 / 55.0$ & 0.0 & 10.0 & 10.0 \\
\hline Rovaniemi & 16 & $50.0 / 50.0$ & $59.4 / 40.6$ & $62.5 / 37.5$ & 17.6 & 5.9 & 23.5 \\
\hline Kuhmo & 23 & $78.3 / 21.7$ & $78.3 / 21.7$ & $80.4 / 19.6$ & 0.0 & 4.3 & 4.3 \\
\hline Evenstad & 26 & $57.7 / 42.3$ & $59.6 / 40.4$ & $59.6 / 40.4$ & 3.8 & 0.0 & 3.8 \\
\hline Total & 249 & $58.8 / 41.2$ & $61.6 / 38.4$ & $65.1 / 34.9$ & 5.0 & 10.1 & 15.1 \\
\hline
\end{tabular}

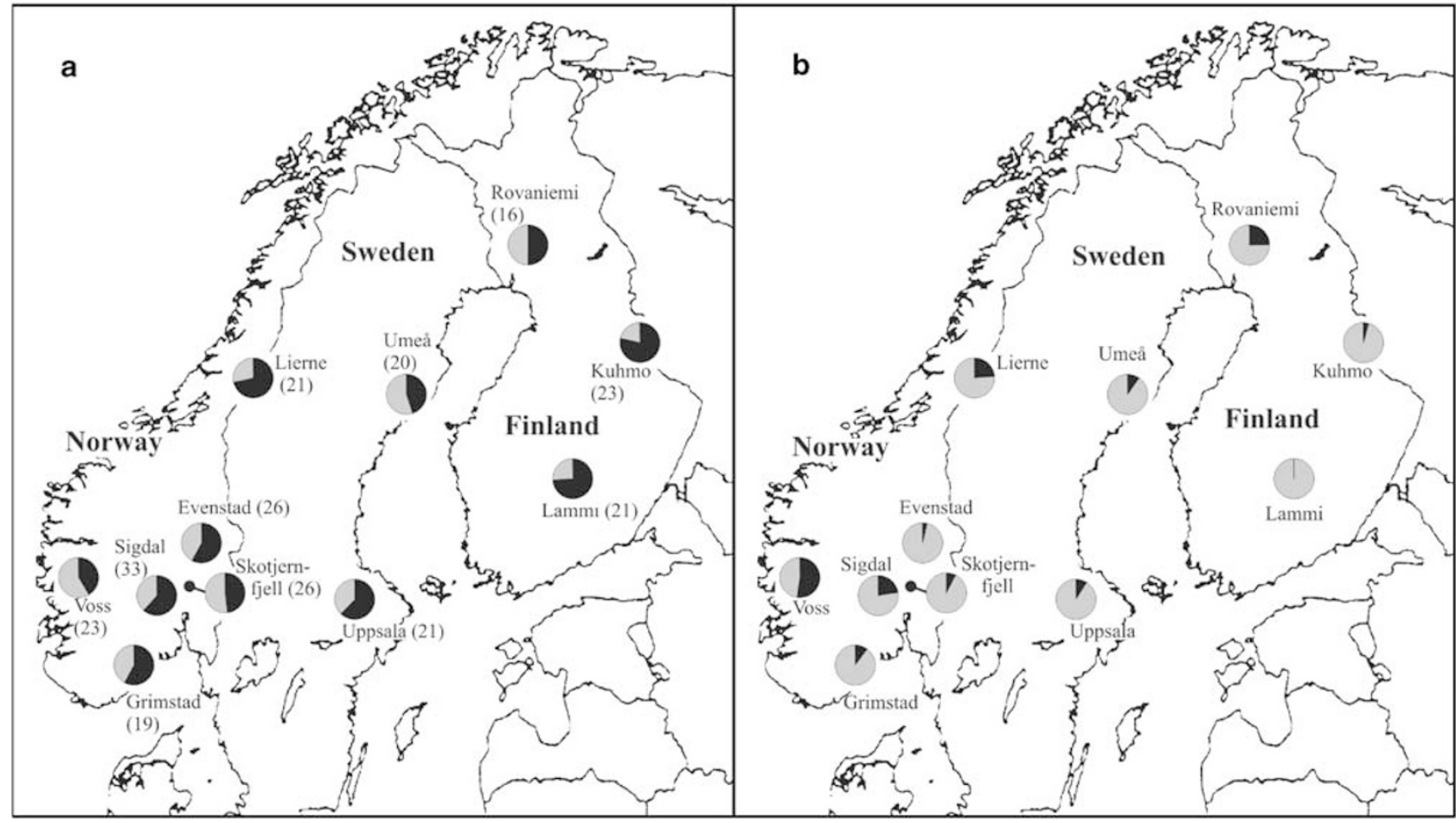

Figure 5 (a) The distribution of $\alpha$ (black section) and $\beta$ (grey section) ITS1 haplotypes in 11 geographic T. abietinum populations. (b) The distribution of nonrecombinant $\alpha$ and $\beta$ nrDNA haplotypes (grey section) and recombinant nrDNA haplotypes (black section) in the populations, including both $\alpha$ and $\beta$ spacer versions.

fruit bodies (TaGu1 and TaGu4) known to possess both ITS1 versions (dikaryons), gave a 1:1 (TaGu1) and 4:3 (TaGu4) segregation of the ITS1 $\alpha$ and ITS1 $\beta$ versions and were consistent with a $1: 1$ ratio $\left(\chi^{2}\right.$ test, $\left.P<0.05\right)$. In order to investigate whether meiotic recombination between $\alpha$ and $\beta$ nrDNA versions had occurred, restriction analyses of ITS2 and the partial IGS2 amplicons from the two spore families, employing the RsaI and DdeI endonucleases, were performed. The ITS2/IGS2-RFLP analyses gave segregation patterns concordant with the ITS1 sequence data, demonstrating cosegregation and a tight linkage between the ITS1/ITS2/IGS2 $\alpha$ and the ITS1/
ITS2/IGS2 $\beta$ spacer types, respectively (data not shown). No meiotic recombinants or progenies that included both $\alpha$ and $\beta$ nrDNA were detected among the 56 monokaryons studied.

\section{Discussion}

This study has shown that there are two fairly divergent types of ribosomal repeat units present in North European populations of $T$. abietinum and that these behave like standard alleles at the population level and in their segregation. Recombination is suppressed within 
them, but does occur within a population-level sample. A third ITS type, recently published by Ko and Jung (2002), has so far not been observed in the North European material.

The Mendelian segregation pattern of the nrDNA variants suggests that the observed intraspecific and sometimes within-individual ITS/IGS nrDNA heterogeneity is caused by divergent nrDNA versions encoded in different nuclei in $T$. abietinum. Several alternative explanations may account for the observed pattern of multiple nrDNA types. The most plausible explanation may be that the nrDNA types evolved independently and diverged some time in the past and were later united in a hybridization event. During divergent speciation, it is predicted that sibling species undergo ITS nrDNA sequence homogenization (Arnheim et al, 1980; Zimmer et al, 1980) and achieve a more or less homogenous ITS sequence distinct from other species. In contrast, speciation via hybridization may lead to a species that harbours divergent, but homologous sequences from the parental lineages.

The fact that the nrDNA spacer sequences (ITS1, ITS2, IGS1 and the partial IGS2) from monokaryons derived from four North European geographic populations all yielded either the $\alpha$ or $\beta$ nrDNA version is suggestive of a past independent evolutionary history of the two European nrDNA lineages. In a recombining lineage with a common evolutionary history, it is to be expected that mutations distribute more or less at random and that particular linkage groups, such as observed in the nrDNA of $T$. abietinum, are nonexistent. The somewhat incongruent topologies of the ITS1 and ITS2 trees suggest that recombination has occurred between different nrDNA lineages. The existence of at least three main ITS1 length types in T. abietinum, where one of the types is shared by the sister taxon T. fusco-violaceum, suggests that T. fuscoviolaceum may have been involved in the evolutionary history of T. abietinum. The ITS1 $\alpha$ type observed in this study was similar in length to an ITS1 type beforehand observed in both North America and Korea, while the $\beta$ type was similar to a unique Korean type (Ko and Jung, 2002). The fact that the North European $\alpha$ and $\beta$ types shared a number of unique polymorphisms not observed in non-European T. abietinum/T. fusco-violaceum specimens indicate that they have recombined and shared a common evolutionary history for some time.

Recently, there have been some reports of ongoing hybridization events in basidiomycetes. Newcombe et al (2000) reported a range of hybrid Melampsora rust genotypes between $M$. medusae and $M$. occidentalis, having ITS versions different from the parental species in both homozygous (one version) and heterozygous (both versions) combinations. A similar pattern has been detected in Flammulina, where a rare natural hybrid contained ITS nrDNA elements from the parental species F. velutipes and F. rossica (Hughes and Petersen, 2000). In Californian populations of H. annosum (Fr.) Bref., two ISGs as well as a hybrid group between the two were detected (Garbelotto et al, 1996). The genetic pattern observed in the geographic T. abietinum populations of Fennoscandia may have a similar explanation, representing a combination of ancient evolutionary divergence in geoisolation as well as more recent genetic recombination of previously isolated populations.

The likelihood of hybridization probably increases when previously geographically isolated populations or taxa that lack strong reproductive barriers come into contact and share the same niche (Brasier, 2000). Three ISGs have been documented in T. abietinum; two in North America which are intersterile, and a third European group which is partially interfertile with the North American groups (Macrae, 1967; Magasi, 1976). Unpublished data suggest that the individuals of the present North European populations all belong to the European ISG. Our data indicate that the European populations may represent a mixture of other lineages that could be intersterile but interfertile with the European populations. Additional mating experiments are needed to explore this topic in more detail.

As an alternative hypothesis, it has been suggested that the extensive ITS1 length variation observed in $T$. abietinum could be a result of gene duplication (Ko and Jung, 2002). In the study of Ko and Jung (2002), only heterokaryotic specimens were examined; thus, whether different nrDNA types were restricted to different nuclei and consequently may represent mere allelic variants, was not evidenced. The Mendelian segregation pattern of ITS types observed in the present study appears to rule out the possibility of paralogous $\alpha$ or $\beta$ nrDNA loci in T. abietinum, at least in the North European populations. It has to be mentioned, though, that paralogous nrDNA have been observed in some fungi ( $\mathrm{O}^{\prime}$ Donnell and Cigelnik, 1997; O'Donnell et al, 1998; Kim and Breuil, 2001), a phenomenon frequently observed in polyploid plant (hybrid) species (eg Sang et al, 1995; Wendel et al, 1995).

The PCR-RFLP analyses of field samples (dikaryons) gave absence or low frequencies of evolutionary intermediate nrDNA types of mixed $\alpha$ and $\beta$ sequences (except for the Voss population). However, recombinant nrDNA types were not observed among the sequenced single spore isolates, probably because of the low number of analysed isolates. The proportion of recombinant nrDNA among populations varied on a spatial scale, which may be attributed to stochastic genetic drift. The Voss population, isolated from the other populations by high mountains, had higher proportion of nrDNA recombinants, which may be attributed to a past founder event. Admittedly, our assignment of $\alpha$ and $\beta$ nrDNA types in dikaryotic field samples was based on digest profiles of the spacer regions, which cannot fully determine whether the nrDNA type is of $\alpha, \beta$ or a recombinant form. Thus, the possibility that some nrDNA variants have been overlooked cannot be ruled out.

The sequence variation observed in the efa and $g p d$ genes, may also well reflect former divergent evolutionary lineages, like the $\alpha$ and $\beta$ nrDNA versions, but these data are less convincing. In isolation, the observed sequence patterns in efa and gpd could be explained by accumulation of mutations over time in a recombining taxon. However, recombination will gradually lead to a breakdown of associations between linked polymorphisms. Hybrids or introgressants are unlikely to be detected when recombination (and homogenization) has occurred over a long period of time, which may be the case regarding efa and $g p d$. Another possibility that cannot be fully dismissed is that genes from one lineage 
have been eliminated after a hybridization event (Wendel et al, 1995).

In the segregation analyses of basidiospore progenies, the $\alpha$ or $\beta$ nrDNA spacer versions cosegregated, and no meiotic recombinants including novel associations of the $\alpha$ and $\beta$ nrDNA were found. This demonstrates that nrDNA is inherited as a single locus in Mendelian fashion, as has also been observed in other basidiomycetes, for example, Laccaria bicolor (Maire) P.D. Orton (Selosse et al, 1996) and Pleurotus cornucopiae (Paulet) Rolland (Iracabal and Labarere, 1994). A low frequency of IGS2 recombinants was observed in L. bicolor (Selosse et al, 1996), while no recombinants was observed in Coprinus cinereus (Schaeff:Fr.) S.F. Gray (Cassidy et al, 1984). The absence of recombinants in the progenies of $T$. abietinum suggests that meiotic recombination within the nrDNA gene family may be suppressed, as observed previously in yeast (Petes and Botstein, 1977). Another possible explanation is that very few linked nrDNA repeats exist. However, nothing is known at present about size and organization of the nrDNA clusters in $T$. abietinum. A higher sample size may possibly have detected meiotic recombinants also in T. abietinum.

As a concluding remark, it must be emphasized that a refined phylogenetic study of worldwide Trichaptum spp. specimens, including nrDNA and other unlinked gene sequences, is needed in order to understand the natural history of T. abietinum and its allies more fully.

\section{Acknowledgements}

This study was supported financially by the University of Oslo (UiO), Norway, Nansenfondene and a scholarship to $\mathrm{H}$ Kauserud from the Research Council of Norway (NFR). The laboratory work was conducted at the Laboratory of Molecular Ecology and Evolution (DNA-lab), and the MycoLab, UiO. We thank C Brochmann and two anonymous reviewers for comments to the manuscript, $G$ Caetano-Anollés for discussion, AC Scheen and KT Hansen for technical assistance, and R Penttila, OJ Sørensen and M Gustafsson for help in connection with the fieldwork. This study is part of $T$ Schumacher's project on population structure and genetic variation in wood-inhabiting basidiomycetes supported by the NFR (grant 125819/410).

\section{References}

Anderson JB, Stasovski E (1992). Molecular phylogeny of northern-hemisphere species of Armillaria. Mycologia 84: 505-516.

Arnheim N, Krystal M, Schmickel R, Wilson G, Ryder O, Zimmer E (1980). Molecular evidence for genetic exchanges among ribosomal genes on nonhomologous chromosomes in man and apes. Proc Natl Acad Sci USA 77: 7323-7327.

Brasier C (2000). The rise of the hybrid fungi. Nature 405: 134135.

Brasier CM, Cooke DEL, Duncan JM (1999). Origin of a new Phytophthora pathogen through interspecific hybridization. Proc Natl Acad Sci USA 96: 5878-5883.

Cassidy JR, Moore D, Lu BC, Pukkila PJ (1984). Unusual organization and lack of recombination in the ribosomal RNA genes of Coprinus cinereus. Curr Genet 8: 607-613.

Felsenstein J (1985). Confidence limits on phylogenies: an approach using the bootstrap. Evolution 39: 783-791.
Franzke A, Mummenhoff K (1999). Recent hybrid speciation in Cardamine (Brassicaceae) - conversion of nuclear ribosomal ITS sequences in statu nascendi. Theor Appl Genet 98: 831-834.

Garbelotto M, Ratcliff A, Bruns T, Cobb FW, Otrosina WJ (1996). Use of taxon-specific competitive-priming PCR to study host specificity, hybridization, and intergroup gene flow in intersterility groups of Heterobasidion annosum. Phytopathology 86: 543-551.

Gilbertson RL, Ryvarden L (1987). North American Polypores, Vol. 2. Megasporaria-Wrightoporia. Fungiflora: Oslo.

Hall TA (1999). BioEdit: a user-friendly biological sequence alignment editor and analysis program for Windows 95/98/ NT. Nucleic Acids Symp Ser 41: 95-98.

Heiman M (1997). Webcutter 2.0. (http://www.firstmarket. com/cutter/cut2.html).

Hughes K, Petersen R (2000). Apparent recombination or gene conversion in the ribosomal ITS region of a Flammulina (Fungi, Agaricales) hybrid. Mol Biol Evol 18: 94-96.

Iracabal B, Labarere J (1994). Restriction site and length polymorphism of the rDNA unit in the cultivated Basidiomycete Pleurotus cornucopiae. Theor Appl Genet 88: 824-830.

Kauserud H, Schumacher T (2001) Outcrossing or inbreeding: DNA markers provide evidence for type of reproductive mode in Phellinus nigrolimitatus. Mycol Res 105: 676-683.

Kim SH, Breuil C (2001). Common nuclear ribosomal internal transcribed spacer sequences occur in the sibling species Ophiostoma piceae and O. quercus. Mycol Res 105: 331-337.

Ko K, Hong SG, Jung HS (1997). Phylogenetic analysis of Trichaptum abietinum based on nuclear 18S, 5.85 and ITS ribosomal DNA sequences. Mycologia 89: 727-734.

Ko KS, Jung HS (2002). Three nonorthologous ITS1 types are present in a polypore fungus Trichaptum abietinum. Mol Phyl Evol 23: 112-122.

Kreuzinger N, Podeu R, Gruber F, Göbl F, Kubicek CP (1996). Identification of some ectomycorrhizal basidiomycetes by PCR amplification of their gpd (glyceraldehyde-3-phosphate Dehydrogenase) genes. Appl Environ Microbiol 62: 3432-3438.

Macrae R (1967). Pairing incompatibility and other distinctions among Hirschioporus [Polyporus] abietinus, H. fusco-violaceus, and H. laricinus. Can J Bot 45: 1371-1398.

Magasi LP (1976). Incompatibility factors in Polyporus abietinus, their numbers and distribution. Mem NY Bot Gard 28: 163173.

Murray MG, Thompson WF (1980). Rapid isolation of high molecular weight plant DNA. Nucleic Acids Res 8: 4321-4325.

Newcombe G, Stirling B, McDonald S, Bradshaw HD (2000). Melampsora $\times$ columbiana, a natural hybrid of M. medusae and M. occidentalis. Mycol Res 104: 261-274.

Nielsen K, Yohalem DS (2001). Origin of a polyploid Botrytis pathogen through interspecific hybridization between Botrytis aclada and B. byssoidea. Mycologia 93: 1064-1071.

O'Donnell K, Cigelnik E (1997). Two divergent intragenomic rDNA ITS2 types within a monophyletic lineage of the fungus Fusarium are nonorthologous. Mol Phyl Evol 7: 103116.

O'Donnell K, Cigelnik E, Nirenberg HI (1998). Molecular systematics and phylogeography of the Gibberella fujikuroi species complex. Mycologia 90: 465-493.

Petes D, Botstein D (1977). Simple Mendelian inheritance of the reiterated ribosomal DNA of yeast. Proc Natl Acad Sci USA 74: 5091-5095.

Ryvarden L, Gilbertson RL (1994). European Polypores. Fungiflora: Oslo.

Sang T, Crawford DJ, Stuessy TF (1995). Documentation of reticulate evolution in peonies (Paeonia) using internal transcribed spacer sequences of nuclear ribosomal DNA: implications for biogeography and concerted evolution. Proc Natl Acad Sci USA 92: 6813-6817. 
Selosse MA, Costa G, Battista CD, Tacon FL, Martin F (1996) Meiotic segregation and recombination of the intergenic spacer of the ribosomal DNA in the ectomycorrhizal basidiomycete Laccaria bicolor. Curr Genet 30: 332-337.

Swofford DL (1999). PAUP* Version. 4.02b. Phylogenetic analysis using parsimony ( ${ }^{*}$ and other methods). Sinauer Associates: Sunderland, MA. Christensen MJ, Latch GCM, Siegel MR, Schadl CL.

Tsai HF, Liu JS, Staben C, Christensen MJ, Latch GCM, Siegel MR, Schadl CL (1994). Evolutionary diversification of fungal endophytes of tall fescue grass by hybridization with Epichlö species. Proc Natl Acad Sci USA 91: 2542-2546.
Wendel JF, Schnabel A, Seelanen T (1995). Bidirectional interlocus concerted evolution following allopolyploid speciation in cotton (Gossypium). Proc Natl Acad Sci USA 92: 280284.

White TJ, Bruns T, Lee S, Taylor J (1990). Amplification and direct sequencing of fungal ribosomal RNA genes for phylogenetics. In: Innis MA, Gelfand DH, Sninsky JJ, White TJ (eds) PCR Protocols: A Guide to Methods and Applications, Academic Press: San Diego, CA.

Zimmer EA, Martin SL, Beverley SM, Kan YW, Wilson AC (1980). Rapid duplication and loss of genes encoding for the $\alpha$ chains of hemoglobin. Proc Natl Acad Sci USA 77: 2158-2162. 